\title{
Saturation Effects in the Magnetic Resonance of Paramagnetic Impurities in Metals
}

\author{
David L. Huber*
}

Physics Department, University of Wisconsin-Madison, Madison, WI 53706, USA

\begin{abstract}
The saturation of the magnetic resonance of paramagnetic impurities in metallic hosts is investigated. The earlier work of N. Bloembergen is extended to metallic films of finite thickness. The absorption line shape and its derivative with respect to the static applied field are calculated. Comparison is made with the corresponding results for an insulating host, and suggestions are given as to the way in which saturation effects might be identified.
\end{abstract}

\section{INTRODUCTION}

Saturation effects in cw (continuous wave) resonance studies of paramagnetic impurities in insulating hosts have been observed and understood for many years. In this case the power absorbed by the magnetic system is given by the standard expression

$$
P=\frac{V \chi_{0} H_{1}^{2} \omega \omega_{0} T_{2}}{1+\left(T_{2} \Delta \omega\right)^{2}+\left(\gamma H_{1}\right)^{2} T_{1} T_{2}}
$$

where $V$ is the volume of the sample, $\chi_{0}$ is the static susceptibility, $H_{1}$ is the amplitude of the oscillating field at frequency $\omega, \omega_{0}$ is the resonance frequency and $\Delta \omega=\omega-\omega_{0}$. The symbol $\gamma$ denotes the gyromagnetic ratio, and $T_{1}$ and $T_{2}$ are the longitudinal and transverse relaxation times, respectively.

The purpose of this letter is to develop and analyze a corresponding expression for the power absorbed by stationary paramagnetic impurities in a conducting medium. Saturation effects in the magnetic resonance of immobile impurities in metallic hosts were first investigated by $\mathrm{N}$. Bloembergen [1]. In this reference, equations were presented for the absorption on resonance $(\Delta \omega=0)$ in both the thin and thick film limits. In this paper we extend Bloembergen's analysis to metallic films of finite thickness and present detailed results for the absorption line shape and the field derivative of the line shape as a function of the static applied field for fixed $\gamma H_{1}\left(T_{1} T_{2}\right)^{1 / 2}$ and as a function of $\gamma H_{1}\left(T_{1} T_{2}\right)^{1 / 2}$ when the system is at resonance.

To the best of our knowledge, there have been no systematic studies of saturation effects associated with the magnetic resonance of paramagnetic impurities in metals. It is hoped that theory presented here will stimulate efforts in this direction.

\section{ANALYSIS}

The essential step in the analysis is the identification of $\left(\partial H_{1} / \partial z\right)^{2}$ as a first integral of Eq. (24) in Ref. [1]. In our investigation of the effects of film thickness, we use a plane wave or 'radar' model in which electromagnetic wave

*Address Correspondence to this Author at the Physics Department, University of Wisconsin-Madison, Madison, WI 53706, USA;

E-mail: huber@src.wisc.edu propagates in a direction normal to the surface of a film of thickness $d$. The amplitude of the magnetic component of the wave at the incident surface is $H_{1}$, and the amplitude at the exit surface is $H_{1} \exp [-d / \delta]$, where $\delta$ denotes the skin depth This being the case, the power absorbed by a metallic film of thickness $d$ can be expressed as the difference between Eq. (25) of [1] evaluated at $z=0$ and the identical expression evaluated $z$ $=d$. We obtain the result

$$
\begin{aligned}
& P=\left(\omega \omega_{0} \chi_{0} T_{2} / 8\right) \delta A\left(1-\Delta \omega T_{2}\right)\left(\gamma^{2} T_{1} T_{2}\right)^{-1} \\
& \times \ln \left[\frac{1+\left(\Delta \omega T_{2}\right)^{2}+\left(\gamma H_{1}\right)^{2} T_{1} T_{2}}{1+\left(\Delta \omega T_{2}\right)^{2}+\left(\gamma H_{1}\right)^{2} T_{1} T_{2} e^{-2 d / \delta}}\right]
\end{aligned}
$$

where $A$ is the area of the film.

In the low-intensity limit, $\left(\gamma H_{1}\right)^{2} T_{1} T_{2} \ll 1$, Eq. (2) becomes

$$
\begin{aligned}
& P=\left(\omega \omega_{0} \chi_{0} T_{2} / 8\right) \delta A\left(1-\Delta \omega T_{2}\right) H_{1}^{2} \\
& \times(1-\exp [-2 d / \delta])\left[1+\left(\Delta \omega T_{2}\right)^{2}\right]^{-1}
\end{aligned}
$$

When $d / \delta>>1$, this expression has the has the form

$$
P=\left(\omega \omega_{0} \chi_{0} T_{2} / 8\right) \delta A\left(1-\Delta \omega T_{2}\right) H_{1}^{2}\left[1+\left(\Delta \omega T_{2}\right)^{2}\right]^{-1}
$$

Equation (4) is in agreement with the results obtained by Dyson in his analysis of conduction electron spin resonance in a thick film in the slow diffusion limit [2,3].

For arbitrary intensities in the thick film limit, Eq. (2) becomes

$$
\begin{aligned}
& P=\left(\omega \omega_{0} \chi_{0} T_{2} / 8\right) \delta A\left(1-\Delta \omega T_{2}\right) \\
& \times\left(\gamma^{2} T_{1} T_{2}\right)^{-1} \ln \left[\frac{1+\left(\Delta \omega T_{2}\right)^{2}+\left(\gamma H_{1}\right)^{2} T_{1} T_{2}}{1+\left(\Delta \omega T_{2}\right)^{2}}\right]
\end{aligned}
$$

while for arbitrary intensities in the thin film limit we find

$$
\begin{aligned}
& P=\left(\omega \omega_{0} \chi_{0} T_{2} / 4\right) d A\left(1-\Delta \omega T_{2}\right) H_{1}^{2} \\
& \times\left[1+\left(\Delta \omega T_{2}\right)^{2}+\left(\gamma H_{1}\right)^{2} T_{1} T_{2}\right]^{-1}
\end{aligned}
$$

In. Eq. (6), it is evident that the absorbed power does not depend explicitly on the skin depth. Nevertheless, the skin depth does enter implicitly in the assumption that the film has finite conductivity. 


\section{DISCUSSION}

In typical paramagnetic resonance experiments, the frequency is held fixed, and the static field is varied. Usually the data are obtained in the derivative mode where $\mathrm{d} P / \mathrm{d} H$ is recorded as a function of the static field $H$ with $\omega$ held constant. In applying the equations, in the preceding section, one makes the transcription $\Delta \omega=-\gamma\left(H-H_{0}\right)=-\gamma \Delta H$ [3]. For narrow lines, $|\Delta \omega| / \omega_{0} \ll<1$, one can replace $\omega_{0}$ by $\omega$ in the prefactor in Eqs. $(2-6)$ thus obtaining an expression that is a function of the static field only through its dependence on $\Delta H$.

In Fig. 1 we show the results for $\mathrm{d} P / \mathrm{d} H$ vs $\gamma \Delta H T_{2}$ for $\gamma H_{1}\left(T_{1} T_{2}\right)^{1 / 2}=0.1,1.0$ and 10 in the thin film limit (Eq. (6)). Fig. 2 shows the corresponding results for a film of intermediate thickness where $d / \delta=1.0$. The numerical data were obtained from Eq. (2). Fig. 3 shows $\mathrm{d} P / \mathrm{d} H$ vs $\gamma \Delta H T_{2}$ in the thick film limit for the same three values of $\gamma H_{1}\left(T_{1} T_{2}\right)^{1 / 2}$ : $0.1,1.0$ and 10 (Eq. (5)). All curves have been normalized to unity at $\Delta H=0$.

Comparing the three figures, it is evident that when $\gamma H_{1}\left(T_{1} T_{2}\right)^{1 / 2} \gg 1$, the effects of saturation are more pronounced in thin films than in thick films, and that in all three cases, the line shape becomes more symmetric with increasing values of this parameter.

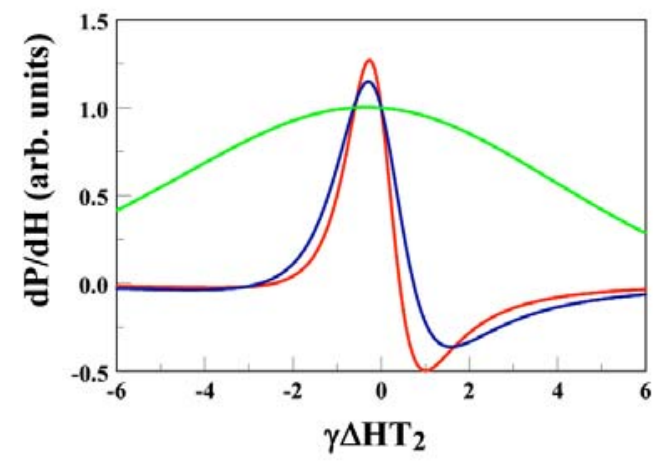

Fig. (1). $\mathrm{d} P / \mathrm{d} H$ (arbitrary units) vs $\gamma \Delta H T_{2}$ in a thin film. The curves are calculated using Eq. (6). (red) $\gamma H_{1}\left(T_{1} T_{2}\right)^{1 / 2}=0.1$; (blue) $\gamma H_{1}\left(T_{1} T_{2}\right)^{1 / 2}=1.0$; (green) $\gamma H_{1}\left(T_{1} T_{2}\right)^{1 / 2}=10$. All curves normalized to 1 at $\Delta H=0$.

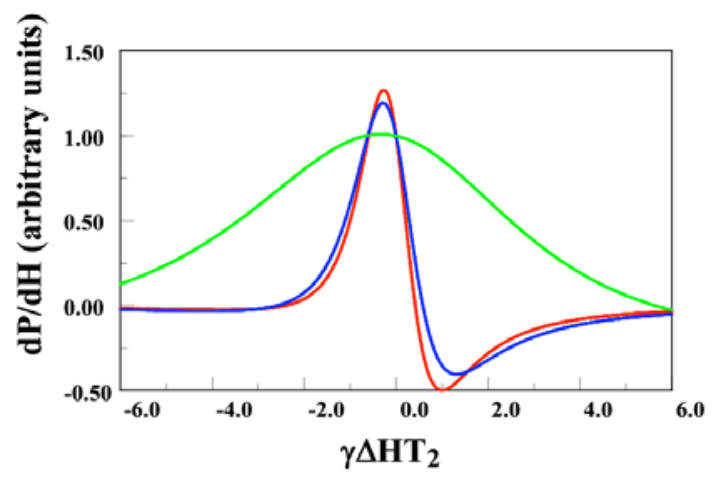

Fig. (2). $\mathrm{d} P / \mathrm{d} H$ (arbitrary units) vs $\gamma \Delta H T_{2}$ in a film of intermediate thickness where $d / \delta=1.0$. The curves are calculated using Eq. (2). (red) $\gamma H_{1}\left(T_{1} T_{2}\right)^{1 / 2}=0.1$; (blue) $\gamma H_{1}\left(T_{1} T_{2}\right)^{1 / 2}=1.0 ;$ (green) $\gamma H_{1}\left(T_{1} T_{2}\right)^{1 / 2}=10$. All curves normalized to 1 at $\Delta H=0$.

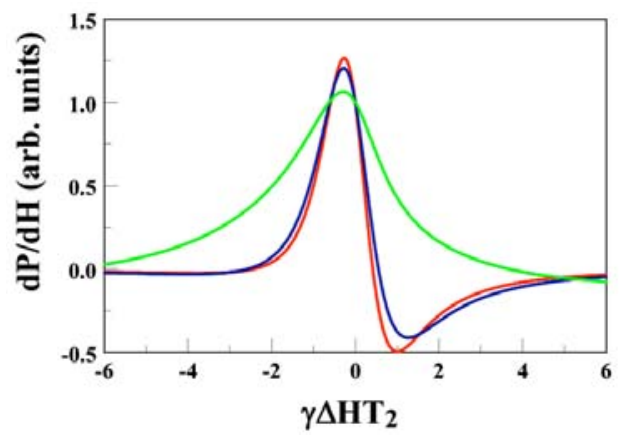

Fig. (3). $\mathrm{d} P / \mathrm{d} H$ (arbitrary units) vs $\gamma \Delta H T_{2}$ in a thick film. The curves are calculated using Eq. (5). (red) $\gamma H_{1}\left(T_{1} T_{2}\right)^{1 / 2}=0.1$; (blue) $\gamma H_{1}\left(T_{1} T_{2}\right)^{1 / 2}=1.0$; (green) $\gamma H_{1}\left(T_{1} T_{2}\right)^{1 / 2}=10$. All curves normalized to 1 at $\Delta H=0$.

As pointed out in Ref. [1], a particularly sensitive technique for detecting saturation effects is to measure the absorption at $\Delta H=0$ divided by $H_{1}^{2}$ as a function of $\gamma H_{1}\left(T_{1} T_{2}\right)^{1 / 2}$. In Fig. 4 we show the results of such a plot for the thin and thick film limits and for the intermediate case $d / \delta=1.0$, with all curves normalized to unity at $H_{1}=0$. From this figure, we see that when $\Delta H=0$, the normalized plots of $H_{1}^{-2} P$ have decreased by $30 \%-50 \%$ when $\gamma H_{1}\left(T_{1} T_{2}\right)^{1 / 2}=1$, thus providing a strong signature of saturation similar to what is seen in insulators.

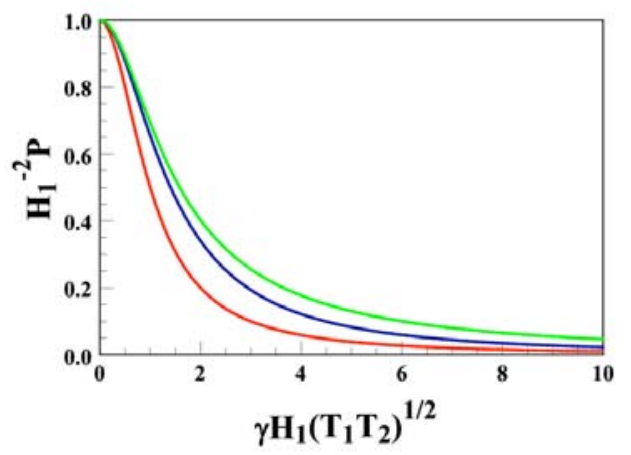

Fig. (4). $H_{1}^{-2} P$ vs $\gamma H_{1}\left(T_{1} T_{2}\right)^{1 / 2}$ with $\Delta H=0$. The blue curve is for $d / \delta$ $=1.0$ The red curve is the limiting result for thin films, $1 /\left(1+x^{2}\right)$, with $x=\gamma H_{1}\left(T_{1} T_{2}\right)^{1 / 2}$, that was obtained in Ref. 1 The green curve is the result for infinitely thick films, $x^{-2} \ln \left(1+x^{2}\right)$, also given in [1].

Note that the plots of $H_{1}^{-2} P$ for finite values of $d / \delta$ are always bounded by the curves for the thick and thin film limits. It should also be mentioned that when $\Delta H=0$, the normalized curves for $H_{1}^{-2} P$ and $H_{1}^{-2} \mathrm{~d} P / \mathrm{d} H$ vs $\gamma H_{1}\left(T_{1} T_{2}\right)^{1 / 2}$ are predicted to be identical for the same value of $d / \delta$.

In summary, we have outlined a theoretical framework for interpreting saturation effects in the magnetic resonance of paramagnetic impurities in metals. The theory, which also applies to nuclear magnetic resonance, interpolates between earlier results [1] for the absorption in the thin film and thick film limits. At this point, there are several comments that need to be made relating to the detection of saturation effects. First, it is necessary that the g-factors of the paramagnetic ions be significantly different from those of the conduction electrons in order to avoid a "spin bottleneck" where the paramagnetic ions resonate in phase with the conduction electrons. This 
means one must have a distinct paramagnetic resonance well separated from the conduction electron resonance. The analysis of the coupled conduction electron spin - paramagnetic ion resonance remains a challenging but distinct problem with progress continuing to be made [4].

Second, the impurity resonance must be homogeneously broadened to avoid the "blurring" of the line shape caused by a distribution of resonance frequencies. As noted, a definitive demonstration of saturation follows from analyzing the absorption on resonance $(\Delta H=0)$. By comparing plots of $H_{1}^{-2} \mathrm{P}$ (or $H_{1}^{-2} \mathrm{~d} P / \mathrm{d} H$ ) vs $H_{1}$ against the theoretical curves (Fig. 4) it will be possible to infer the values of the product $T_{1} T_{2}$. Since $T_{2}$ can be extracted from the resonance line shape in the unsaturated regime, one can determine the value of $T_{1}$.

\section{ACKNOWLEDGEMENTS}

The author thanks Carlos Rettori for stimulating his interest in resonance saturation and for providing helpful comments on the research.

\section{REFERENCES}

[1] Bloembergen N. On the magnetic resonance absorption in conductors. J Appl Phys 1952; 23: 1383-1389.

[2] Dyson FJ. Electron spin resonance in metals II theory of electron spin diffusion and the skin effect. Phys Rev 1955; 98: 349-359.

[3] Feher G, Kip AF. Electron spin resonance in metals I experimental. Phys Rev 1955; 98: 337-349.

[4] Fokina NP, Khutsishvili KO, Atsarkin, VA. ESR and longitudinal response in metals containing localized paramagnetic centers with spin S > 1/2. Appl Mag Res 2003; 24: 197-213. 